\title{
GLP1R agonists: primary cardiovascular prevention and oral administration
}

GLP1R

agonists afford cardiovascular superiority even in primary prevention

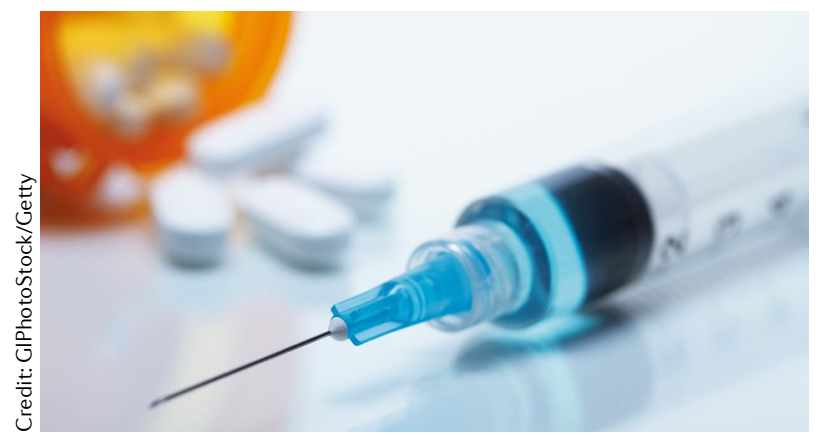

a weekly subcutaneous injection of dulaglutide $(1.5 \mathrm{mg})$ or placebo. The mean age was 66.2 years, median $\mathrm{HbA}_{1 \mathrm{c}}$ level was $7.2 \%$ and $46.3 \%$ of participants were women.

During follow-up (median 5.4 years), the primary end point (first occurrence of nonfatal myocardial infarction, nonfatal stroke or death from cardiovascular disease, including unknown causes) occurred in $12.0 \%$ of the dulaglutide group and $13.4 \%$ of the placebo group (HR 0.88 , 95\% CI 0.79-0.99, $P=0.026$ ).

All-cause mortality did not differ significantly between the two groups. Of note, gastrointestinal events were significantly more common with dulaglutide than with placebo (47.4\% versus $34.1 \%$; $P<0.0001$ ).

"The broad inclusion criteria, high proportion of women, and the representativeness of the recruited participants in REWIND suggest that dulaglutide might be effective for both primary and secondary cardiovascular prevention in a high proportion of patients with type 2 diabetes," comment the trial investigators. "We now have evidence from the DECLARE-TIMI 58 and REWIND trials that [sodium/ glucose cotransporter 2] SGLT2 inhibitors and GLP1R agonists afford cardiovascular superiority even in primary prevention, with SGLT2 inhibitors preventing heart failure and GLP1R agonists preventing atherosclerotic events, and both potentially affording renal protection," add Verma, Mazer and Perkovic in a Comment article accompanying the trial publication in The Lancet.

A potential barrier to the clinical uptake of GLP1R agonists is that all the currently approved versions are administered by subcutaneous injection. Therefore, semaglutide has been developed in an oral tablet form, and the cardiovascular safety of oral semaglutide was tested in the PIONEER 6 trial. A total of 3,183 patients with type 2 diabetes and at high cardiovascular risk were randomly assigned to receive oral semaglutide or placebo. During follow-up (median 15.9 months), the primary outcome (first occurrence of death from cardiovascular causes, nonfatal myocardial infarction or nonfatal stroke) occurred in 3.8\% of patients receiving oral semaglutide and $4.8 \%$ of patients receiving placebo (HR 0.79, 95\% CI 0.57-1.11, $P<0.001$ for noninferiority).

Furthermore, in the PIONEER 4 trial, once-daily oral semaglutide was compared with once-daily subcutaneous injection of liraglutide or placebo in 711 patients with type 2 diabetes. Compared with subcutaneous liraglutide, oral semaglutide was noninferior in decreasing $\mathrm{HbA}_{1 \mathrm{c}}$ levels and superior in decreasing body weight at 26 weeks. "Use of oral semaglutide could potentially lead to earlier initiation of GLP1R agonist therapy in the diabetes treatment continuum of care," conclude the investigators.

Gregory B. Lim

ORIGINAL ARTICLES Gerstein, H. C. et al. Dulaglutide and cardiovascular outcomes in type 2 diabetes (REWIND): a double-blind, randomised placebo-controlled trial. Lancet https://doi.org/10.1016/S0140-6736(19)31149-3 (2019)| Husain, M. et al. Oral semaglutide and cardiovascular outcomes in patients with type 2 diabetes. N. Engl.J. Med. https://doi.org/10.1056/ NEJMoa1901118 (2019) | Pratley, R. et al. Oral semaglutide versus subcutaneous liraglutide and placebo in type 2 diabetes (PIONEER 4): a randomised, double-blind, phase 3a trial. Lancet https://doi.org/10.1016/S0140-6736(19)31271-1 (2019)

FURTHER READING Nassif, M. \& Kosiborod, M. Effect of glucose-lowering therapies on heart failure. Nat. Rev. Cardiol. 15, 282-291 (2018) 